Research Paper

\title{
Pre-operative Serum Alkaline Phosphatase as a Predictor for Hypocalcemia Post-Parathyroid Adenectomy
}

\author{
Seng Cheong Loke ${ }^{1 凶}$, Alvin Wai Kit Tan ${ }^{2}$, Rinkoo Dalan², Melvin Khee-Shing Leow ${ }^{2,3}$ \\ 1. Institute of Gerontology (IG), Universiti Putra Malaysia, Malaysia; \\ 2. Tan Tock Seng Hospital (TTSH), Singapore; \\ 3. Singapore Institute for Clinical Sciences (SICS), Singapore
}

Corresponding author: Dr Seng Cheong Loke. Institute of Gerontology, Universiti Putra Malaysia, 43400 Serdang, Selangor Darul Ehsan, MALAYSIA. E-mail: lokesengcheong@gmail.com.

(c) Ivyspring International Publisher. This is an open-access article distributed under the terms of the Creative Commons License (http://creativecommons.org/ licenses/by-nc-nd/3.0/). Reproduction is permitted for personal, noncommercial use, provided that the article is in whole, unmodified, and properly cited.

Received: 2012.07.12; Accepted: 2012.09.10; Published: 2012.09.18

\begin{abstract}
Background. Post-operative hypocalcemia (POH) may complicate parathyroidectomy for primary hyperparathyroidism. This study investigates the relationship between $\mathrm{POH}$ and pre-operative risk factors to identify a simple method to predict $\mathrm{POH}$ risk.

Methods. Retrospective data on risk factors for 29 patients was collected for age, pre-operative serum calcium, alkaline phosphatase (ALP), parathyroid hormone (PTH), adenoma size, gender, and bisphosphonate pre-treatment. These were screened to exclude those with small effect sizes, and analyzed using Univariate General Linear Modeling (GLM) with trough serum calcium (TSC) as the dependent variable. The regression function of the significant variables against TSC was plotted with $95 \% \mathrm{Cl}$ fit lines. The cut-off regression value was read from the lower fit line for the threshold TSC of $2.0 \mathrm{mmol} / \mathrm{L}$.

Results. After screening, log-transformed age $(r=0.600)$, ALP $(r=-0.415)$, and PTH $(r=-0.433)$ were entered into GLM analysis, which showed that only ALP was significant $(p=0.016$ Eta-squared $=0.220$ ). The GLM model had a partial Eta-squared of 0.559 with $98 \%$ observed power. The plot of TSC against log-ALP gave an ALP cut-off of $340 \mathrm{U} / \mathrm{L}$.

Conclusions. The study shows that there is a strong relationship between ALP and TSC, and that patients with a pre-operative ALP less than $340 \mathrm{U} / \mathrm{L}$ are unlikely to have symptomatic $\mathrm{POH}$ (100\% sensitivity, $95 \%$ specificity). While vitamin D was not analyzed in this study, the ALP cut-off is conservative and should still screen out cases with severe vitamin $D$ deficiency. We therefore recommend that pre-operative ALP be utilized to complement clinical protocols for $\mathrm{POH}$ management in parathyroid adenomectomy patients.
\end{abstract}

Key words: Alkaline Phosphatase, Hypocalcemia, Linear Models, Primary Hyperparathyroidism, Surgery

\section{INTRODUCTION}

$\mathrm{POH}$ is a well known complication following parathyroid adenectomy for PHP, and this can be either transient or persistent. Persistent hypocalcemia is usually caused by surgical damage to the remaining parathyroid glands. In its chronic state, this may be completely asymptomatic, or may manifest as cataracts, dry skin, brittle nails and poor dentition.
Transient hypocalcemia is more commonly found, complicating $12 \%$ of parathyroidectomies for PHP $[1,2]$. Acutely, this results in neuromuscular irritability which can present as numbness, paresthesia, muscular twitching, and cramps. In serious cases, stridor, bronchospasm, cardiac arrhythmias, angina, cardiac failure, syncope, and 
seizures may occur. Symptomatic POH seldom manifests unless TSC falls below the threshold of 2.0 $\mathrm{mmol} / \mathrm{L}(8.0 \mathrm{mg} / \mathrm{dL})$ [3].

It is thought that transient hypocalcemia is related to the acute fall in PTH which causes a reduction in serum calcium and a corresponding increase in serum phosphate. In some cases where prolonged hyperparathyroidism induces a state of high bone turnover, the fall in PTH may result in unopposed bone deposition of calcium and phosphate, causing HBS with low levels of both serum calcium and phosphate [1]. While HBS is associated with more severe and prolonged $\mathrm{POH}$, these two conditions do not always occur together.

As the consequences of significant $\mathrm{POH}$ are potentially serious, it is clinically useful if we have some means of reliably predicting its occurrence so that appropriate preventive protocols can be devised. Brasier's study had demonstrated that certain factors such as age, pre-operative serum calcium, ALP, PTH, and adenoma size, affect the likelihood of developing HBS [1].

While Brasier's study proposed four predictive variables for HBS, their use of a discriminant function combining these variables may prove too unwieldy for routine clinical practice. If a simpler yet reliable alternative for predicting symptomatic $\mathrm{POH}$ could be found, this would indeed be useful to clinicians.

A pilot study conducted by the authors suggested that there was an inverse relationship between pre-operative serum ALP and post-operative TSC, and that this association was strongest among the variables assessed in Brasier's study. In addition, no significant difference was found between patients who received bisphosphonate pre-treatment and those who did not [4].

This is interesting as some case reports suggest that $\mathrm{POH}$ can be attenuated by bisphosphonate pre-treatment, and this finding was supported by another Taiwanese study $[5,6]$. However, the effect of bisphosphonates on bone turnover and remineralisation after surgery in PHP is presently unknown, and its use cannot be routinely recommended in all cases.

The primary objective of the study was to model the relationship between the pre-operative risk factors described in Brasier's study and post-operative TSC, and to determine the strength of that relationship. The secondary objective was to select the risk factors with the strongest influence on post-operative TSC, and derive a simple threshold function below which few patients would be expected to develop significant $\mathrm{POH}$.

\section{MATERIALS AND METHODS}

The study was approved by the National Healthcare Group Domain Specific Review Board (DSRB-C/06/278) and the Malaysian Ministry of Health Research Ethics Committee (KKM/NIHSEC/08/0804/P07-132) in accordance with current guidelines on Good Clinical Practice and the Declaration of Helsinki. Data was collected retrospectively based on patient records. As this was done anonymously, informed consent was waived by the ethics review boards.

The study population consisted of patients operated for PHP at two tertiary institutions (Tan Tock Seng Hospital, Singapore; Kuala Lumpur General Hospital, Malaysia) over the period from 1997 to 2007. Only patients diagnosed with PHP (ICD-9 Code 252.0) with a single adenoma subsequently removed surgically were included. Those with multiple lesions were excluded as they had significantly higher risk of permanent hypoparathyroidism after surgery. Additional exclusion criteria were those treated with drugs which can affect calcium metabolism, particularly oestrogens, selective oestrogen receptor modulators, and vitamin $\mathrm{D}$ derivatives, with bisphosphonates being an important exception. This is because unlike other confounders, bisphosphonates form an integral part of hypercalcemia management in PHP and are frequently used. In addition, we also planned in this study to examine the effect of this factor on the development of $\mathrm{POH}$. Patients younger than 16 years and pregnant women were excluded due to higher physiological ALP levels. Patients with stage 2 chronic kidney disease and above with a calculated creatinine clearance less than $60 \mathrm{~mL} / \mathrm{min} / 1.73 \mathrm{~m}^{2}$ were excluded as this could affect calcium and phosphate metabolism.

Standard ALP (reference interval: 32-103 U/L, Synchron LX20 \& UniCel DxC, Beckman Coulter, Fullerton, California; Roche-Hitachi 747, Roche Diagnostics, Basel, Switzerland) was measured without isoenzyme determination, and included only if done in the six month period prior to surgery. The PTH (reference interval: 0.5-5.1 pmol/L, electrochemiluminescent assay, Elecsys 1010 analyser, Roche Diagnostics; Immulite 2000, DPC Biermann, Bad Nauheim, Germany) value used was the most recent reading taken prior to surgery. TSC (reference interval: $2.15-2.55 \mathrm{mmol} / \mathrm{L}, \quad 8.60-10.20 \mathrm{mg} / \mathrm{dL}$, Beckman Coulter Synchron LX20, UniCel DxC, Roche-Hitachi 747) was taken to be the lowest calcium level corrected for serum albumin using Payne's method over the 72 hour period after completion of 
parathyroidectomy [7]. A longer monitoring period was not deemed necessary as most patients' parathyroid function recover within 30 hours of surgery, with subsequent improvement in serum calcium [8]. Data for patient age at operation, PTH, and peak pre-operative serum calcium corrected for albumin was also collected. Adenoma volume was estimated on ultrasonography, or from the histology report of the surgical specimen when available.

\section{Statistical Analysis}

The risk factors from Brasier's study which we examined were age, pre-operative serum calcium, ALP, PTH, and adenoma size. In addition, we also looked at the effect of gender and bisphosphonate pre-treatment.

For the primary objective, risk factors were first screened to select those with the strongest influence on TSC. This was done using bivariate testing with Pearson's correlation for continuous variables, and an unpaired T-test for categorical variables. Curve fitting against TSC was done for continuous variables to determine whether logarithmic transformation was required to improve linearity, and normality was checked using a Kolmogorov-Smirnov test. Logarithmic transformation was performed if the variable was not normally distributed but could be normalized by transformation, or if the variance explained by the variable improved by more than $20 \%$ after transformation. The cut-off for inclusion was set as a moderate effect size, with Pearson's $r>0.3$ for bivariate correlation and Cohen's $\mathrm{d}>0.6$ for the unpaired T-test [9]. The included variables were then analyzed with GLM using continuous variables as covariates, categorical variables as fixed factors, and TSC as the dependent variable.

For the secondary objective, outliers were screened using a plot of Cook's against Mahalanobis distances. A plot of the regression function for the significant variables against TSC was made along with $95 \% \mathrm{CI}$ fit lines. The value for the regression function was then read from the lower fit line for the threshold TSC of $2.0 \mathrm{mmol} / \mathrm{L}(8.0 \mathrm{mg} / \mathrm{dL})$.

All statistical computations were performed using SPSS for Windows version 19.0 (SPSS Inc, Chicago, Illinois, USA), and conducted at $5 \%$ level of significance.

\section{RESULTS}

Case records for 106 patients with ICD-9 Code 252.0 (PHP) were screened, of which 29 satisfied the inclusion and exclusion criteria. Most of these patients were excluded as they were either managed medically, or wrongly coded for PHP. Patient demographics were as follows: age (17-81 years); male $(n=13)$, female $(n=16)$; Chinese $(n=17)$, Malay $(n=8)$, Indian $(n=4)$; bisphosphonate pre-treatment $(n=15)$, no pre-treatment $(\mathrm{n}=14)$. Relevant pre-operative factors are listed in Table 1 . All three patients with a TSC less than the threshold of $2.0 \mathrm{mmol} / \mathrm{L}(8.0$ $\mathrm{mg} / \mathrm{dL}$ ) had clinical symptoms of hypocalcemia, which was persistent in only one patient (case \#9), while none of those with a TSC $>2 \mathrm{mmol} / \mathrm{L}(8.0$ $\mathrm{mg} / \mathrm{dL}$ ) were symptomatic.

TABLE I. Relevant pre- and post-operative factors ( $N=$ 29)

\begin{tabular}{|c|c|c|c|c|c|c|}
\hline \multicolumn{6}{|c|}{ Pre-op. } & \multirow{2}{*}{$\begin{array}{l}\text { Post-op. } \\
\text { TSC } \\
(\mathrm{mmol} / \mathrm{L})\end{array}$} \\
\hline No & Age & $\begin{array}{l}\text { ALP } \\
(\mathrm{U} / \mathrm{L})\end{array}$ & $\begin{array}{l}\text { PTH } \\
(\mathrm{pmol} / \mathrm{L})\end{array}$ & $\begin{array}{l}\text { Peak Ca } \\
(\mathrm{mmol} / \mathrm{L})\end{array}$ & $\begin{array}{l}\text { Adenoma } \\
\text { Vol (mL) }\end{array}$ & \\
\hline Mean & 51.9 & 273 & 43.9 & 3.17 & 2.82 & 2.28 \\
\hline SD & 18.0 & 480 & 49.3 & 0.43 & 4.40 & 0.28 \\
\hline 1 & 35 & - & 20.2 & 3.14 & 1.94 & 2.43 \\
\hline 2 & 52 & 232 & 181.0 & 4.62 & 6.64 & 2.30 \\
\hline 3 & 50 & - & 7.5 & 3.25 & 0.84 & 2.43 \\
\hline 4 & 66 & - & 11.4 & 2.62 & - & 2.27 \\
\hline 5 & 65 & - & 13.7 & 3.33 & 0.58 & 2.73 \\
\hline 6 & 57 & - & 5.4 & 2.68 & 0.12 & 2.36 \\
\hline 7 & 80 & 84 & 14.1 & 2.85 & 0.27 & 2.60 \\
\hline 8 & 61 & 135 & 14.1 & 3.18 & 0.34 & 2.57 \\
\hline 9 & 17 & 2464 & 153.0 & 2.99 & 2.55 & 1.70 \\
\hline $10^{*}$ & 22 & 400 & 118.0 & 3.41 & 1.96 & 1.57 \\
\hline 11 & 67 & 84 & 47.1 & 3.19 & 0.84 & 2.60 \\
\hline 12 & 28 & 198 & 18.2 & 2.98 & 0.13 & 2.00 \\
\hline 13 & 81 & 89 & 9.1 & 3.07 & 0.52 & 2.54 \\
\hline $14^{*}$ & 63 & 440 & 94.6 & 3.34 & 2.98 & 1.76 \\
\hline 15 & 73 & 159 & 43.6 & 3.48 & 0.52 & 2.46 \\
\hline 16 & 77 & 223 & 14.4 & 2.91 & 0.05 & 2.09 \\
\hline 17 & 44 & 174 & 10.5 & 2.95 & 0.07 & 2.36 \\
\hline 18 & 41 & 244 & 54.1 & 3.13 & 2.62 & 2.23 \\
\hline 19 & 72 & 120 & 11.2 & 3.09 & 14.14 & 2.35 \\
\hline 20 & 35 & 126 & 67.7 & 2.78 & 2.11 & 2.16 \\
\hline 21 & 53 & 72 & 14.0 & 3.15 & 1.13 & 2.53 \\
\hline 22 & 59 & 228 & 49.9 & 3.58 & 18.85 & 2.13 \\
\hline 23 & 36 & 150 & 12.0 & 2.94 & 2.57 & 2.24 \\
\hline 24 & 55 & 137 & 90.0 & 4.14 & 7.85 & 2.73 \\
\hline 25 & 55 & 110 & 12.2 & 3.00 & 2.20 & 2.33 \\
\hline 26 & 30 & 64 & 19.5 & 2.82 & 0.35 & 2.18 \\
\hline 27 & 45 & 77 & 14.8 & 2.87 & 2.38 & 2.29 \\
\hline 28 & 24 & 456 & 139.0 & 3.59 & - & 2.17 \\
\hline 29 & 61 & 85 & 12.0 & 3.02 & 1.57 & 2.09 \\
\hline
\end{tabular}

Serum calcium normalized after 72 hours 
For the primary objective, curve fitting suggested that logarithmic transformation was needed for age, ALP, and PTH, but not for the other variables. Bivariate testing selected log-age $(r=0.600$, $\mathrm{p}=0.001), \log$-ALP $(\mathrm{r}=-0.415, \mathrm{p}=0.025)$, and log-PTH $(\mathrm{r}=-0.433$, $\mathrm{p}=0.019)$, while excluding pre-operative serum calcium $(\mathrm{r}=0.121, \mathrm{p}=0.548)$, adenoma size $(\mathrm{r}=-0.067, \mathrm{p}=0.741)$, gender $(\mathrm{d}=0.338, \mathrm{p}=0.376)$, and bisphosphonate pre-treatment $(\mathrm{d}=0.136, \mathrm{p}=0.719)$. Normality was confirmed for log-age $(p=0.462)$, $\log$-ALP $(p=0.603), \log -\mathrm{PTH} \quad(\mathrm{p}=0.115)$, and TSC $(p=0.873)$. GLM analysis showed that only log-ALP $(p=0.016$ Eta-squared $=0.220)$ was a significant factor, while log-age $(p=0.064$ Eta-squared $=0.123)$ and $\log$-PTH $\quad(p=0.484 \quad$ Eta-squared $=0.016) \quad$ were non-significant. The GLM analysis model had an overall partial Eta-squared (analogous to R-squared from multiple linear regression) of 0.559 with an observed power of $98 \%$.

For the secondary objective, the single risk factor with the strongest influence on post-operative TSC from GLM analysis was ALP. The plot of Cook's against Mahalanobis distances shows a clear outlier (case \#9) (Fig. 1). On reviewing the case records, case \#9 was found to be an case of PHP with severe concurrent vitamin D deficiency (25-hydroxyvitamin $\mathrm{D}<12.5 \mathrm{nmol} / \mathrm{L}$ [5 ug/L]) which elevates ALP disproportionately due to the element of secondary hyperparathyroidism accentuating the background primary hyperparathyroidism. After removing this outlier, a scatter plot of TSC against log-ALP was made, giving an ALP value of $340 \mathrm{U} / \mathrm{L}$ for the threshold TSC of $2.0 \mathrm{mmol} / \mathrm{L}(8.0 \mathrm{mg} / \mathrm{dL})$ from the lower fit line (Fig. 2).

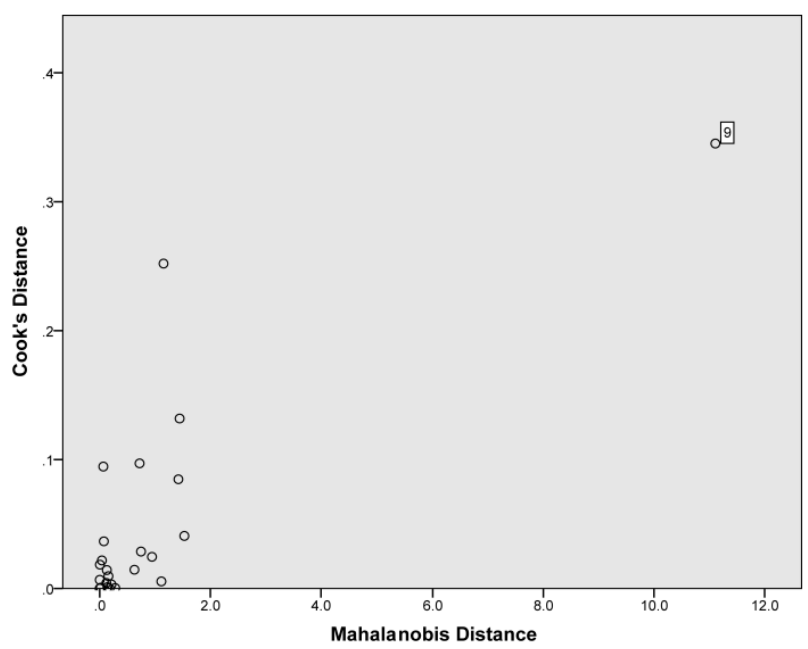

FIG. I. Plot of Cook's against Mahalanobis distances to detect outliers for the regression function of trough post-operative serum calcium against log pre-operative ALP.

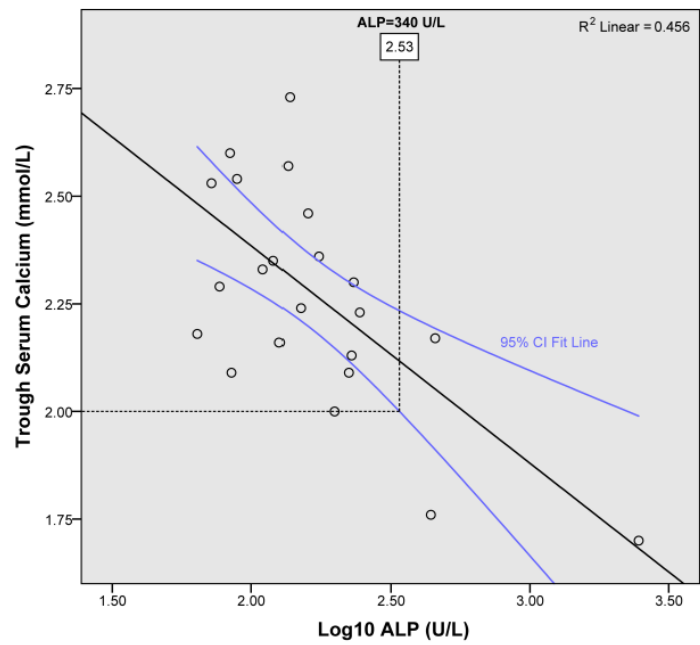

FIG. 2. Scatterplot of trough post-operative serum calcium against log pre-operative ALP, with $95 \%$ confidence interval fit lines.

\section{DISCUSSION}

For the primary objective, the three risk factors that emerged from initial screening were log-age, log-ALP, and log-PTH, but only log-ALP remained significant after GLM analysis. The effect size for log-ALP was large (Eta-squared $=0.220$ ) while the effect sizes for log-age and log-PTH were medium (Eta-squared $=0.123$ ) and small (Eta-squared $=0.016$ ) respectively [9]. Log-age just missed achieving significance $(p=0.064)$, and this was likely due to the relatively small sample size. The GLM model itself was rigorous, with all variables normally distributed and accounting for $56 \%$ of the variance in TSC.

For the secondary objective, while it would have been reasonable to include both age and ALP for the analysis, ALP alone was used so as to simplify the development and application of the threshold function. The 95\% CI fit lines represent the uncertainty in the regression function, and the lower fit line provides a conservative estimate of ALP for the TSC threshold. Exclusion of the outlier (case \#9) was justified because of the large effect it has on the regression function (Fig. 1), and the clinical picture of severe vitamin D deficiency which can inflate PTH and hence ALP values in PHP [10]. Moreover, this portion of the analysis was intended to model the relationship between ALP and TSC in most typical cases of PHP so as to obtain a reliable ALP cut-off value.

From the analysis, this means that patients with a pre-operative ALP less than $340 \mathrm{U} / \mathrm{L}$ have a low likelihood of developing a TSC less than $2.0 \mathrm{mmol} / \mathrm{L}$ $(8.0 \mathrm{mg} / \mathrm{dL})$, and are hence unlikely to have 
symptomatic $\mathrm{POH}$. In this study, all three subjects who developed a TSC less than the threshold and experienced symptomatic POH had ALP values greater than $340 \mathrm{U} / \mathrm{L}$. Only one subject with a serum ALP value greater than $340 \mathrm{U} / \mathrm{L}$ had an uneventful post-operative course, giving a sensitivity of $100 \%$ and specificity of $95 \%$.

The use of standard ALP without isoenzyme determination increases the likelihood of a spurious result from a non-parathyroid cause. However, bone-specific ALP is not readily available in every institution, and in practice does not pose a significant problem as the source of ALP excess is usually obvious. When paired with gamma-glutamyl transpeptidase in a standard liver panel, a hepatic cause for raised ALP can usually be excluded. In any case, additional post-operative precautions should not increase the risk for these patients.

In adults, the liver and bone isoenzymes form a large part of the serum ALP pool, while the intestinal isoenzyme is found in small amounts $(<20 \%$ total ALP) in a minority of patients (about 20\%) [11]. The presence of intestinal ALP in serum is dependent on $\mathrm{ABO}$ status, and is found most frequently in subjects with blood groups $\mathrm{O}$ and $\mathrm{B}$, especially after a fatty meal [12]. While it would be desirable to know the $\mathrm{ABO}$ status, variation in ALP levels caused by the intestinal isoenzyme can be minimised by taking the sample in the fasting state.

The large effect size for log-ALP suggests that high pre-operative bone turnover is linked with significant $\mathrm{POH}$ risk. The large covariance between ALP and PTH (Pearson's r $=0.671, p<0.001$ ) means that either variable could theoretically be used for determination of a threshold value. However, in practice ALP is better as a predictor as it gives more stable readings compared with PTH due to the big difference in biological half-lives ( 8 hours and 4 minutes respectively), and this is supported by the GLM analysis which selected ALP and excluded PTH $[13,14]$.

The relationship between ALP and TSC should be further explored because of the strong correlation between them. It has previously been shown that in PHP, markers of bone formation and resorption were raised compared with normal controls. After parathyroidectomy, bone resorption markers fell significantly within 24 hours while bone formation markers were unchanged $[15,16]$. This suggests that osteoclast activity becomes decoupled from osteoblast activity in the immediate post-operative period, perhaps due to an acute fall in PTH. A direct effect of this decoupling is the rapid drop in serum calcium that characterises $\mathrm{POH}$. The duration of hypocalcemia is limited by speedy recovery of parathyroid function in the remaining glands, and seldom exceeds three days unless the other glands were compromised during surgery or HBS is present [8]. As ALP is a marker of bone formation, a higher pre-operative level gives rise to greater decoupling between bone formation and resorption after parathyroidectomy, thus increasing the risk of significant $\mathrm{POH}$.

Although the difference in TSC between patients with and without bisphosphonate pre-treatment was not significant, it is difficult to draw a conclusion from this as the study was not adequately powered for this purpose. This contrasts with the previously mentioned Taiwanese study by Lee et al. which was able to find a significant difference with fewer $(n=23)$ patients [6]. As the literature is unclear on the effect of bisphosphonate pre-treatment on $\mathrm{POH}$, further work could be done in this area.

The main weakness in this study is the small sample size. However, this is sufficient for purposes of the GLM analysis given the high observed power for the model $(98 \%)$. While a larger sample size could have given a narrower confidence interval for the regression and hence a higher cut-off value for ALP, this is not a major problem as the current cut-off of 340 $\mathrm{U} / \mathrm{L}$ already screens out $85 \%$ of patients, with high sensitivity and specificity for $\mathrm{POH}$.

Vitamin D levels were not available for most subjects in this study, and this may be an issue as long-standing patients with coexistant PHP and severe vitamin $\mathrm{D}$ deficiency are especially prone to $\mathrm{POH}$. However, these patients have markedly elevated levels of PTH and ALP, and should hence be picked up when screening based on an ALP cut-off $[17,18]$. Even in this study, the outlier with severe vitamin D deficiency which was excluded from the analysis (case \#9) had a grossly elevated ALP (Table 1). Hence the ALP cut-off of $340 \mathrm{U} / \mathrm{L}$ which was based on typical cases of PHP would still pick up those unusual cases with coexistant severe vitamin D deficiency.

In conclusion, the study shows that patients with a pre-operative ALP less than $340 \mathrm{U} / \mathrm{L}$ are unlikely to have symptomatic post-operative $\mathrm{POH} \quad(100 \%$ sensitivity, $95 \%$ specificity). While vitamin $\mathrm{D}$ was not analyzed in this study, the ALP cut-off is conservative and should still screen out cases of PHP with severe vitamin $\mathrm{D}$ deficiency. Serum calcium monitoring can be done less frequently and for a shorter duration in low-risk patients, facilitating early discharge and reduced resource utilisation. We therefore recommend that pre-operative ALP be utilized to complement clinical protocols addressing the management of $\mathrm{POH}$ in parathyroid adenomectomy 
patients.

\section{ACKNOWLEDGEMENTS}

The authors would like to thank Dr David Ng from the Singapore General Hospital Department of Nuclear Medicine and PET, and Dr Andrew Loy at the Tan Tock Seng Hospital ENT Department for assistance with patient tracking.

\section{COMPETING INTERESTS}

The authors have declared that no competing interest exists.

\section{REFERENCES}

1. Brasier AR, Nussbaum SR. Hungry bone syndrome: clinical and biochemical predictors of its occurrence after parathyroid surgery. Am J Med. 1988; 84: 654-60. doi:0002-9343(88)90100-3 [pii].

2. Zuberi KA, Urquhart AC. Serum PTH and ionized calcium levels as predictors of symptomatic hypocalcemia after parathyroidectomy. Laryngoscope. 2010; 120 Suppl 4: S192. doi:10.1002/lary.21656.

3. Loke SC, Kanesvaran R, Yahya R, Fisal L, Wong TW, Loong YY. Efficacy of an intravenous calcium gluconate infusion in controlling serum calcium after parathyroidectomy for secondary hyperparathyroidism. Ann Acad Med Singapore. 2009; 38: 1074-80.

4. Loke SC, Chew EK, Dalan R. A retrospective analysis of hungry bone syndrome in post-operative parathyroidectomy patients. In: Proceedings of the 12th International Congress of Endocrinology. International Congress of Endocrinology (ICE). Lisbon, Portugal: Medimond; 2004.

5. Kumar A, Ralston SH. Bisphosphonates prevent the hungry bone syndrome. Nephron. 1996; 74: 729.

6. Lee IT, Sheu WH, Tu ST, Kuo SW, Pei D. Bisphosphonate pretreatment attenuates hungry bone syndrome postoperatively in subjects with primary hyperparathyroidism. J Bone Miner Metab. 2006; 24: 255-8. doi:10.1007/s00774-005-0680-x.

7. Payne RB, Little AJ, Williams RB, Milner JR. Interpretation of serum calcium in patients with abnormal serum proteins. Br Med J. 1973; 4: 643-6.

8. Brasier AR, Wang CA, Nussbaum SR. Recovery of parathyroid hormone secretion after parathyroid adenomectomy. J Clin Endocrinol Metab. 1988; 66: 495-500

9. Cohen J. Statistical Power Analysis for the Behavioral Sciences. L Erlbaum Associates; 1988.

10. Moosgaard B, Christensen SE, Vestergaard P, Heickendorff L, Christiansen P, Mosekilde L. Vitamin D metabolites and skeletal consequences in primary hyperparathyroidism. Clin Endocrinol (Oxf). 2008; 68: 707-15. doi:CEN3109 [pii] 1111/j.1365-2265.2007.03109.x.

11. Van Hoof VO, Hoylaerts MF, Geryl H, Van Mullem M, Lepoutre LG, De Broe ME. Age and sex distribution of alkaline phosphatase isoenzymes by agarose electrophoresis. Clin Chem. 1990; 36: 875-8.

12. Langman MJ, Leuthold E, Robson EB, Harris J, Luffman JE, Harris H. Influence of diet on the "intestinal" component of serum alkaline phosphatase in people of different $\mathrm{ABO}$ blood groups and secretor status. Nature. 1966; 212: 41-3.

13. Pickkers P, Snellen F, Rogiers P, Bakker J, Jorens P, Meulenbelt J, et al. Clinical pharmacology of exogenously administered alkaline phosphatase. Eur J Clin Pharmacol. 2009; 65: 393-402. doi:10.1007/s00228-008-0591-6.

14. Bieglmayer C, Prager G, Niederle B. Kinetic analyses of parathyroid hormone clearance as measured by three rapid immunoassays during parathyroidectomy. Clin Chem. 2002; 48: 1731-8.

15. Guo CY, Holland PA, Jackson BF, Hannon RA, Rogers A, Harrison BJ, et al. Immediate changes in biochemical markers of bone turnover and circulating interleukin-6 after parathyroidectomy for primary hyperparathyroidism. Eur J Endocrinol. 2000; 142: 451-9. doi:1420451 [pii].

16. Grey A, Mitnick MA, Shapses S, Ellison A, Gundberg C, Insogna K. Circulating levels of interleukin-6 and tumor necrosis factor-alpha are elevated in primary hyperparathyroidism and correlate with markers of bone resorption--a clinical research center study. J Clin Endocrinol Metab. 1996; 81: 3450-4.
17. Adler JT, Sippel RS, Chen H. 25-hydroxyvitamin D status does not affect intraoperative parathyroid hormone dynamics in patients with primary hyperparathyroidism. Ann Surg Oncol. 2010; 17: 2958-62. doi:10.1245/s10434-010-1125-x.

18. Ozbey N, Erbil Y, Ademoglu E, Ozarmagan S, Barbaros U, Bozbora A. Correlations between vitamin D status and biochemical/clinical and pathological parameters in primary hyperparathyroidism. World J Surg. 2006; 30: 321-6. doi:10.1007/s00268-005-0239-y. 